\title{
How well do Humans Capture the Sounds of Speech in Writing?
}

\author{
Nora Turoman, Suzy J. Styles* \\ Division of Psychology, School of Humanities and Social Sciences, \\ Nanyang Technological University, Singapore \\ *suzy.styles@ntu.edu.sg
}

\begin{abstract}
A large body of research on connections between sensory modalities has shown that deep connections exist between sound and vision, such that people have a tendency to associate certain sounds with certain visual properties, including line-drawn shapes. While recognising the role of written language in audio-visual associations, previous research has largely considered written language a potential source of bias rather than a means of gaining deeper insights into underlying audio-visual associations. We looked to ancient and unfamiliar writing systems spanning recorded human history, to explore whether humans have tried to encode certain characteristics of speech sounds in the letters they created to represent them. Our findings have revealed that modern humans can correctly identify unfamiliar letters at levels higher than would be obtained by chance, and that scripts which encode a particular sound with a particular set of visual characteristics tend to have more correct guesses. This suggests that humans share certain correspondences between sound and sight, which transcend both geographical space and historical time. The present multisensory demonstration aims to provide an interactive experience of the powerful connection between sounds and written letters through a series of activities integrating vision, audition, touch and imagination.
\end{abstract}

Keywords: Sound symbolism, cross-modal processing, evolution of language

\section{Experimental investigation}

In the field of linguistic sound symbolism, there has been growing recognition that humans tend to match particular speech sounds to particular visual shapes (e.g., Sapir, 1929; Köhler, 1929; Ramachandran \& Hubbard, 2001). Previous research has regarded soundshape associations in writing as a potential source of bias. We have, in a series of experiments, found letters to be a fertile site for investigating links between vision and audition.

In three experimental investigations, participants saw pairs of ancient and unfamiliar letters representing /i/ (the 'ee' vowel in 'feet') and $/ u /$ (the 'oo' vowel in 'shoe') derived from a systematic review of 'The World's Writing Systems' (Daniels \& Bright, eds., 1996).

Results showed that modern participants were able to correctly guess the identities of ancient and unfamiliar letters at levels above chance. We also found that scripts with the highest numbers of correct guesses encoded certain sounds using certain visual characteristics (e.g., curvature, width, complexity and enclosure for $/ u /$ ) while scripts with the lowest numbers of correct guesses did the opposite (e.g., straightness, tallness, simplicity, less enclosure for /u/) (c.f. D'Onofrio, 2014). See Figure 1.

The findings suggest that humans over geographic space and historical time have encoded something about the sounds of speech in the letters they choose to represent them, and that humans share correspondences between vision and audition which transcend space and time (Turoman \& Styles, in preparation). 


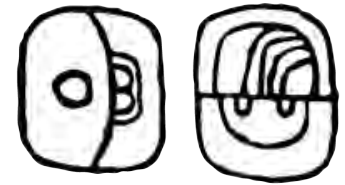

"Which one of these Ancient Mayan glyphs represents the sound 'oo'?"

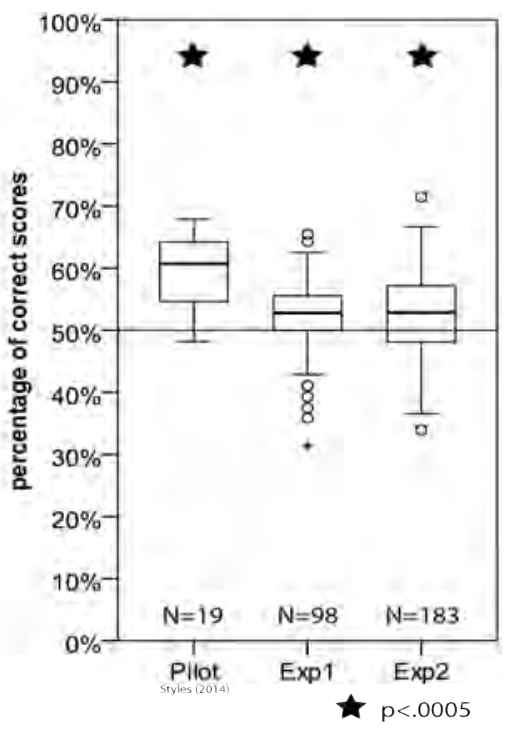

Figure 1. Example question and results

\section{A demonstration}

To provide an experience of the relationships between vision and audition encoded in writing systems of the world, we created two interactive multisensory demonstrations at Si15 Soundislands Festival.

Letter guessing game. Gallery visitors were invited to guess which letters represent which sounds in ancient and unfamiliar scripts, in an activity derived from the experiments mentioned above. Tactile objects depicting contrasting letter forms on opposite surfaces will be placed in the demonstration space, and visitors will be invited to arrange the tokens according to the sounds they think 'go best' with the letters. Depicted letter pairs included some of the most and least 'guessable' scripts obtained in the original experiments (above), allowing visitors' decisions to be compared with previously collected responses. Completed arrangements were compared with an answer key on-the-spot.

Collaborative glyph generation. Gallery visitors were be invited to take part in a collabora- tive glyph generation exercise, in which they can created their own letters, by drawing novel shapes that they feel best represented particular speech sounds.

The participants listened to a series of audio recordings of the following vowel sounds: /i/ (the 'ee' vowel in 'feet'), /u/ (the 'oo' vowel in 'shoe'), /a/ (the 'ah' vowel in 'arm'), and the high front rounded vowel $/ y /$, often written as ü. This sound does not exist in English, but occurs in a number of world languages including German, French, Mandarin and Cantonese. Participants wore headphones, and followed an onscreen prompt to draw a novel glyph they feel best represents each of the vowels, on coloured paper pads.

Glyph creations were collated in a wall display at the festival, as part of a growing installation of sound-shape symbolism. They can be viewed at the Brain Language and Intersensory Perception (BLIP) Lab website, URL http://blogs.ntu.edu.sg/blip.

\section{Acknowledgements}

Supported by a Nanyang Assistant Professorship Grant to SJS: The Shape of Sounds in Singapore.

\section{References}

Daniels, P. T., \& Bright, W. (Eds.). (1996). The World's Writing Systems. Oxford: Oxford University Press.

D'Onofrio, A. (2014). Phonetic Detail and Dimensionality in Sound-shape Correspondences: Refining the Bouba-Kiki Paradigm. Psychological Science, 57, 367-393.

Köhler, W. (1929). Gestalt psychology. New York: Liveright Publishing Corporation.

Ramachandran, V. S., \& Hubbard, E. M. (2001). Synesthesia - a window into perception, thought and language. Journal of Consciousness Studies, 8, 123-34.

Styles, S. J. (2014). 'What can ancient and unfamiliar scripts tell us about sound symbolism?' International Multisensory Research Forum, June 2014, Amsterdam.

Sapir, E. (1929). A study in phonetic symbolism. Journal of Experimental Psychology, 12, 225-239. 